\title{
Optical Identification of Gamma-Ray Bursts at Kenting Observatory
}

\author{
Wei-Hsin Sun \\ National Central University, Chung-Li, Taiwan 320, ROC \\ Shun-Tang Tseng \\ National Central University, Chung-Li, Taiwan 320, ROC
}

\begin{abstract}
A remotely-controlled observing station, the Kenting Observatory, has been established in the Kenting National Park, at the southern tip of Taiwan. There are four observing systems at the Observatory: (1) a Meade 16-inch reflector with an SBIG spectrograph plus an SBIG STV; (2) an RC-16inch reflector with an FLI CCD; (3) a Celestron 14-inch with an ST-7E CCD; and (4) a Binocular system of two Canon F1.8 telephoto lens with two FLI CCDs. One of the scientific tasks of this observatory is to provide fast response to satellite alerts in the hopes of identifying the optical counterparts of Gamma-Ray Bursts (GRBs) via the global effort of GRB Coordinated Network (GCN). In this conference proceedings, we describe the instruments and the science projects being carried out in the Observatory.
\end{abstract}

\section{Introduction}

The Kenting Observatory (hereafter KTO) is located in the National Museum of Marine Biology and Aquarium (NMMBA). The Museum is situated in CherCheng Village in the Heng-Chun Peninsula of Ping-Dong County, about $18 \mathrm{~km}$ north to the Kenting area. The location of NMMBA belongs to the Kenting National Park, which governs a large area of the Southern tip of Taiwan.

The Observatory is established for the purpose of providing the universities and high schools in Taiwan a major observing facility that could be accessed remotely through Internet, in view of the many advantages that remote observation could offer. However, in addition to the educational and promotional purposes, KTO also provides a platform for many scientifically significant research projects. Because the Observatory is located in a National Museum at sea-level, the night sky transparency and darkness are not superb. Nevertheless, these factors do not prevent the Observatory from doing projects of discovery type, such as the search for optical counterparts of Gamma-Ray Bursts (GRBs). Below we describe briefly the telescopes and associated equipments of the Observatory, and then present the scientific attempts. 


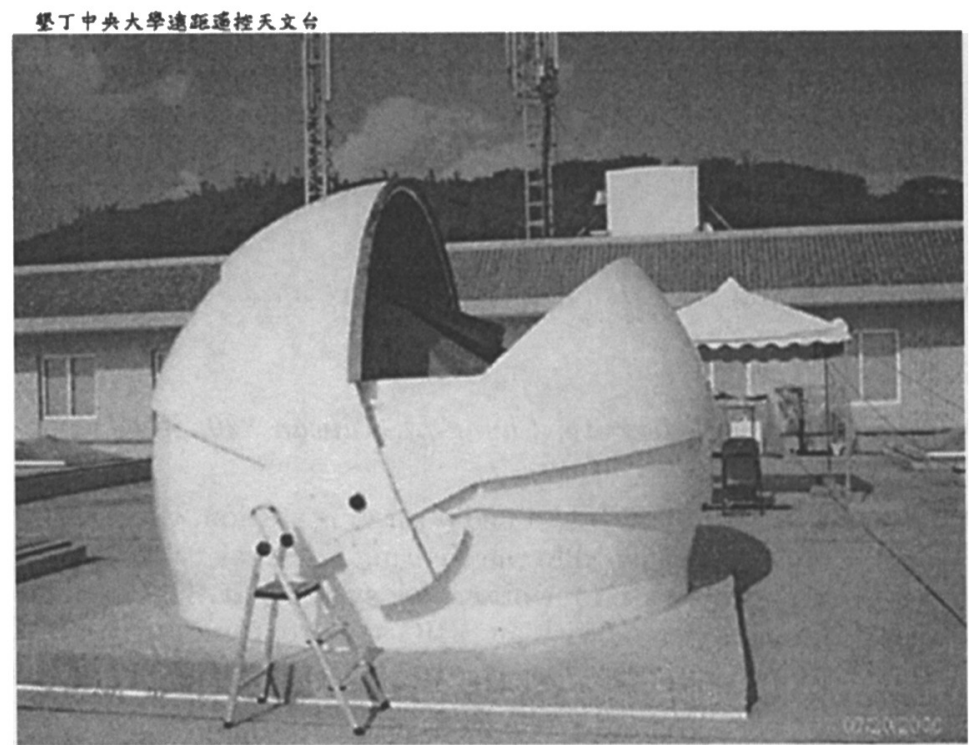

Figure 1. The AstroHaven clam-shell dome at the Kenting Observatory. The two halves open to the North and the South, respectively, and can stop at desired angle, providing effective wind-shielding.

\section{The Observatory and the Equipments}

The remote observing project is funded by the Ministry of Education (MOE), the National Science Council (NSC), and the National Central University (NCU). The NMMBA was very generous and willing to support KTO with an office space, power, and network connection. We thus decided to set the Observatory in NMMBA, to save the funding and effort of developing a new site.

The first observing instrument of the Observatory, the Meade 16-inch telescope, was erected on May 4th, 2000, which was protected by simple tent before the actual dome was constructed. The dome arrived in late June. It is a clamshell type enclosure and is made by AstroHaven in Canada (Figure 1). It has two halves and can open one side or both sides to any desired opening angles. This provides effective wind shielding, which is especially important in the winter when the gusty North-Eastern seasonal wind prevails in Heng-Chun Peninsula. We have implemented a number of modifications before this dome is in full operation and behaves satisfactorily. These modifications include tighter rain-proof sealing, more reliable wire-control for the open and close functions of the shells instead of wireless-control, revised latch-stop, and air-conditioning.

This clam-shell type of dome, which does not use shutter mechanism, offers a great convenience in terms of the equipment it could accommodate. More than one telescope can be placed in the dome, as long as they do not interfere 


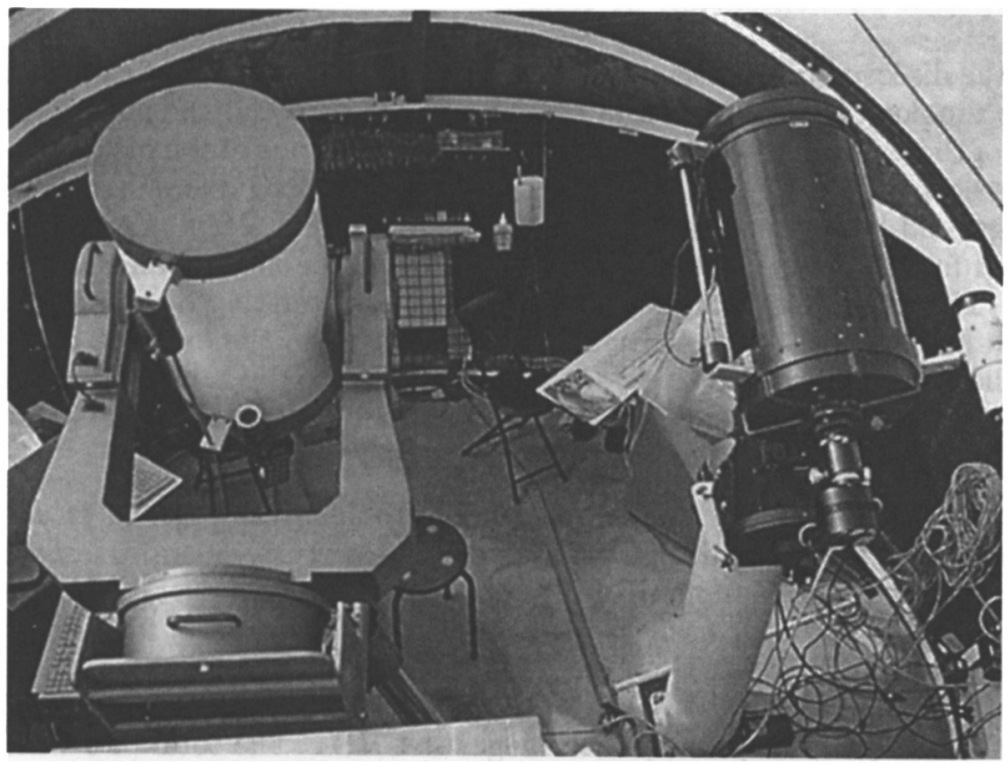

Figure 2. There are two telescopes side by side in the dome, a Meade 16-inch on a custom-made equatorial mount (left) and a Celestron 14inch on a Paramount GT-1100 (right).

with each other. Currently there are two telescopes in our dome side by side, a Meade LX200 16-inch and a Celestron 14-inch (Figure 2).

The Meade 16-inch is equipped with an SBIG low resolution spectrograph. We have removed the slit so the system can perform slitless spectroscopy. The Celestron 14-inch has an ST-7E attached, with the built-in color filter wheel using standard broad-band filters. Each telescope/CCD system is controlled by a Pentium II level computer system. Aside from the control computers, there is one more computer in the dome dedicated to receiving satellite alerts from the GCN.

In addition to the above instruments, there is another telescope of RitcheyChretien type with an aperture of 16-inch. This telescope will be mounted on a GT-1100ME equatorial mount and may replace the C-14 once the RC-16 is ready. We also set up a binocular type of system which consists of two Canon $200 \mathrm{~mm}$ F1.8 telephoto lens. This system will be used to carry out a narrow band imaging survey of Northern sky in several wavelength ranges of major emission lines.

Both remote control and satellite linkage need fast and reliable network connection. Chinese Telecom provides the Observatory with a dedicated T1 line from NMMBA to Ping-Dong University of Science and Technology, where the $\mathrm{T} 1$ line joins the TANET, the academic backbone supported by MOE. 


\section{Optical Identification of GRBs}

Since the discovery of GRBs in the early 70's and the subsequent confirmation of their cosmological distribution in the 90's, the nature and the emission mechanisms of these powerful celestial objects have become one of the major challenges in high energy astrophysics (van Paradijs et al. 2000 and references therein). In 1997, ground-based observations prompted by satellite alerts (e.g. BeppoSAX) revealed for the first time the optical counterpart of a GRB (van Paradijs et al. 1997). This is an important step forward in understanding how and where the large amount of energy is generated. However, due to the extreme difficulty of immediate response to satellite alerts and the fast fading of the optical afterglows (OAs), there have only been a handful (about two dozens) of GRBs whose optical afterglows were recorded.

The success of ROTSE observations of GRB990123 (Akerlof et al. 1999) has stimulated the inauguration of many small (aperture $~ 50 \mathrm{~cm}$ ) telescopes that are connected to the Gamma- Ray Bursts Coordinated Network (GCN, see the web site: http://gcn.gsfc.nasa.gov/gcn/ for further references) for satellite alert and related information. However, the best example in the search of optical counterpart, the GRB990123, has put forth stringent requirements on the systems which hope to acquire optical images of GRBs. The total of 6 white light images acquired between $22 \mathrm{sec}$ and $10 \mathrm{~min}$ by ROTSE after the initial gammaray event showed that the estimated $V$ magnitude of the OA dropped from 8.9 to 14.3. This fast fading nature asks for prompt acquisition and exposure within a few minutes of the burst, or the OA will fade and not be detected by small telescopes.

Thus to cope with this rigorous requirement, we have arranged that the telescopes at Kenting Observatory, while performing other observational projects, are always at standby mode for the response to the satellite alerts. Currently we receive GCN notices in the email mode, which means that the alerts are in ordinary email format (time delay: $5-30 \mathrm{sec}$ ). In the near future, we will collaborate with HETE-2 project and convert the receiving mode to socket control (time delay: $0.1-2.0 \mathrm{sec}$ ) .

Fast response is essential for it will deliver the information on the temporal behavior at the early stage of the phenomenon, but it is not enough. Spectral evolution, on the other hand, provides much more information on how the postburst shock evolves (Sari et al. 1998). We thus set up the GRAB project - Gamma-Ray Alerted Binoculars at KTO for these scientifically challenging tasks. The scientific goals of the GRAB project are two-folded: to obtain multiwavelength as well as multi-epoch observations of GRBs.

The GRAB project at first included two F1.8 telephoto lens, as in the ROTSE project, to cope with the large positional uncertainty of alerts from CGRO. However, as the CGRO was de-commissioned and as HETE-2 comes in as replacement, the positional accuracy has been improved significantly, from degrees down to arcminutes suitable for target acquisition with ordinary long focal length telescopes. We thus reassign the scientific missions for the F1.8 binoculars, and bring in Meade-16inch and C-14inch (with RC-16inch later) for the GRAB project. In short, the Binoculars have developed from two 11-cm to 


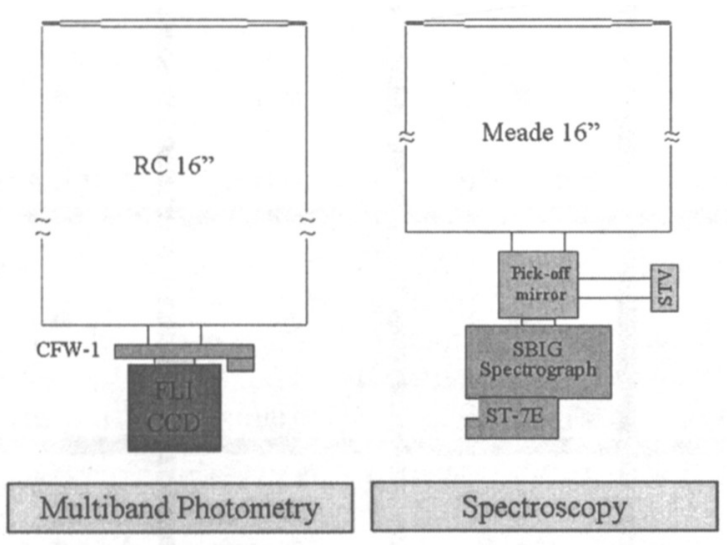

Figure 3. This schematic diagram shows the observing algorithm of the GRAB project. The RC-16 (now C-14) takes multi-color photometry of the target field while the Mead-16 obtains the slitless spectroscopy.

two 40-cm tubes. The C-14 (eventually RC-16) is equipped with direct imaging CCD plus standard broad-band filters. While the Meade- 16 will take the slitless spectra of the target field (Figure 3). Practises and drills show that we could obtain the first 10-sec exposure of the HETE-2 alert field in 2.5 minutes.

\section{An Example of Other Science Projects at KTO - Asteroid Occul- tation}

KTO, with observing staff present all the time, offers a high probability of coverage of astronomical events. Asteroid occultations are scientifically important events that happen all year round. Right before this meeting, in the evening of January 3rd, 2001, we observed an event of the asteroid 12Victoria, with a diameter of $117 \mathrm{~km}$, occulting a background star SAO92745, from UT 11:52 to 13:39. The star is bright, at about 8th magnitude. To our surprise, the asteroid is only 3 magnitude less and can be seen directly in the sequence of images depicting the progress of the event (Figure 4). We will provide the observations to the IOTA for record and will perform analysis on the following issues: the brightness variation of the asteroid, the exact time of occultation, and the orbit determination. These information will allow us to learn more about the rotation of the asteroid and help refine the orbit prediction models.

\section{Conclusions}

With the help of MOE, NSC, NCU, and NMMBA, we have set up a remotely controlled observatory in the Kenting area. One of the major scientific goals is to provide fast response to the satellite alerts in order to obtain optical observations, 


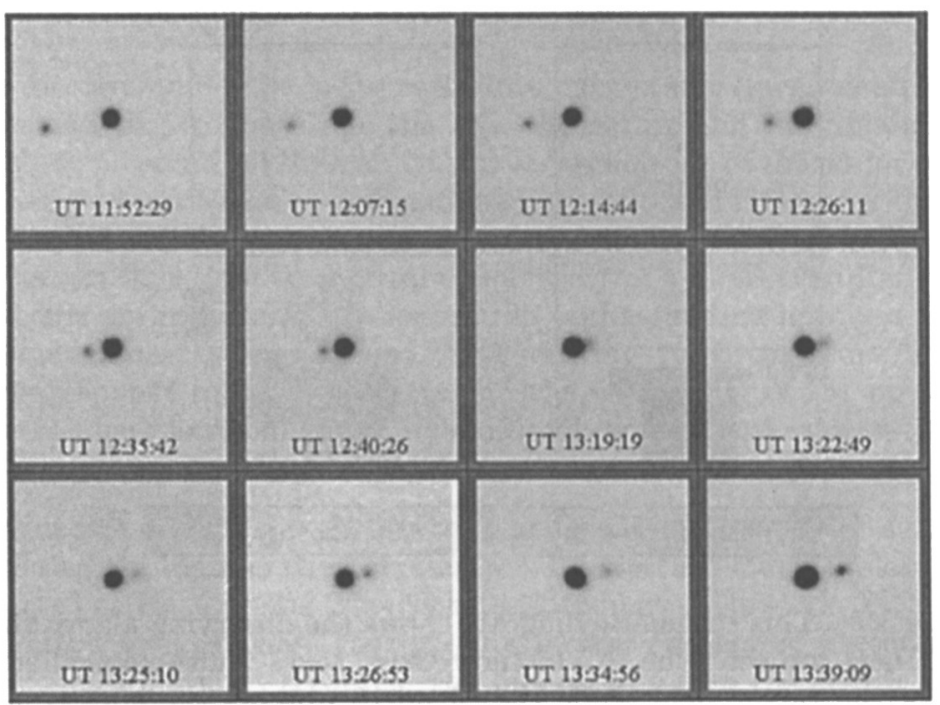

Figure 4. KTO observed an occultation event in the evening of January 3rd, 2001. From UT11:52 to 13:39, the asteroid 12Victoria moved from left to right, and occulted the background star SAO92745 for a brief moment. The star is at about 8.3 magnitude while the asteroid is 3 magnitudes fainter. Both objects are clearly seen in the images.

both temporal and spectral, for the optical counterparts of GRBs. The observing system has been completed and has been in standby mode for satellite alerts. Additional projects, such as asteroid occultation, are also being carried out with preliminary results obtained and being analysed.

Acknowledgments. The authors thank the support of NMMBA for the logistic support and thank Chinese Telecom for the dedicated T1 line. We thank Dr. Claudia Lemme for providing vital information of asteroid occultation events. We also thank Prof. Jing-Yao Hu, Drs. Xiao-Jun Jiang, Yu-Lei Qiu, and Jan-Yan Wei (BAO) for the help in the observation of the occultation event. This project is supported by the grants of MOE 89070250, NSC 89-2515-S-008003, NSC 89-2515-S-008-005, and NSC 89-2515-S-008-006.

\section{References}

Akerlof et al. 1999, Nature, 398, 400.

Sari 1998, ApJ, 494, L49.

van Paradijs et al. 1997, Nature, 386, 686.

van Paradijs et al. 2000, Ann.Rev.Astro.\&Astrophys., Vol.38, 379. 